\title{
Angle-Resolved Electron Scattering from $\mathrm{H}_{2} \mathrm{O}$ near $0^{\circ}$
}

\author{
R. Kadokura, ${ }^{1}$ A. Loreti, ${ }^{1}$ Á. Kövér, ${ }^{2}$ A. Faure, ${ }^{3}$ J. Tennyson, ${ }^{1}$ and G. Laricchia ${ }^{1}$ \\ ${ }^{1}$ UCL Department of Physics and Astronomy, University College London, Gower Street, London WC1E 6BT, United Kingdom \\ ${ }^{2}$ MTA Institute for Nuclear Research (Atomki), Bem ter 18/c, H-4026 Debrecen, Hungary \\ ${ }^{3}$ Université Grenoble Alpes, 621 avenue Centrale, 38400 Saint-Martin-d'Hères, France
}

(Received 17 March 2019; revised manuscript received 30 April 2019; published 16 July 2019)

\begin{abstract}
An electron beam, characterized by a high-angular discrimination $\left(\simeq 0.7^{\circ}\right)$, has been used to measure the total (elastic plus inelastic) cross section of $\mathrm{H}_{2} \mathrm{O}$ in the energy range 3-100 eV. Broad coincidence is found with recent experiments, including a pronounced shoulder in the 6-12 eV region. However, at energies $\lesssim 6 \mathrm{eV}$, the present cross sections are $\simeq 30 \%$ higher. Furthermore, forward scattering has been probed in the angular range $0^{\circ}-3.5^{\circ}$ and measures of the average (rotationally and vibrationally summed) differential elastic cross sections for incident energies $\leq 12 \mathrm{eV}$ are obtained at a scattering angle $\simeq 1^{\circ}$. The measurements, which provide the first test of theoretical predictions in an angular region experimentally unexplored until now, are found to be within 1 standard deviation of corresponding $a b$ initio $R$-matrix calculations.
\end{abstract}

DOI: 10.1103/PhysRevLett.123.033401

Interactions between electrons and water are of fundamental importance in our Universe and to life as it is known (e.g. [1-5]). Over the years, investigations of electron interactions with $\mathrm{H}_{2} \mathrm{O}$ have produced a considerable compendium of experimental and theoretical data, the scope of which extends beyond advancing basic knowledge to include studies of planetary atmospheres, the interstellar medium, and medicine (e.g., Refs. [6-11]). For instance, the propagation of electrons in biological tissue, composed mainly of water, has been found to lead to dangerous DNA lesions (single- and double-DNA breakages) [12], requiring accurate determinations of $e^{-}+\mathrm{H}_{2} \mathrm{O}$ cross sections for modeling electron diffusion in living matter $[8,9,11]$.

Prior to the present Letter, convergence among measurements of the total cross section $\left(\sigma_{T}\right)$ for $e^{-}+\mathrm{H}_{2} \mathrm{O}$ scattering remained poor partly due to the variable angular discrimination $(\theta)$ against forward elastic scattered particles (FESP) of each experiment, typically in the range $0.5-2^{\circ}$. This is because the permanent dipole moment of the $\mathrm{H}_{2} \mathrm{O}$ molecule $(1.854 \mathrm{D})$ results in distant (low-angle) scattering so that the detection of FESP can be significant (e.g., Refs. $[13,14])$, greatly affecting the determinations of $\sigma_{T}$ (e.g., Refs. [15-18]). The effect of FESP has also prevented, until now, measurements of the differential elastic scattering cross section $\left(d \sigma_{e l} / d \Omega\right)$ at any angle smaller than $10^{\circ}$, hindering direct comparisons with theoretical descriptions in a crucially sensitive angular region [19-21].

For example, dipolar molecules are calculated to have large rotational excitation cross sections, which are important in astronomical applications (e.g., Ref. [22]). These cross sections are dominated by the low angle scattering and thus far have all been supplied by theory. However, on the theoretical side, difficulties arise from the nonconvergence of the partial wave expansion in the fixed-nuclei (FN) approximation due to the long-range nature of the electron-dipole interaction. The procedure, widely adopted to deal with this issue and often referred to as the Born closure formula, is to employ the dipolar Born approximation (BA) to obtain the cross section for the high partial waves and add it to that for low partial waves computed in the FN approximation to account for short-range effects (e.g., Refs. $[23,24])$. However, at low energies, the low-angle scattering described by the BA contribution totally dominates the cross section. As a consequence, comparisons with experiments are difficult and attempts to correct measurements by using the same approximation or various extrapolation procedures render the comparison inconclusive (e.g., Refs. [22,25]).

In this Letter, we report cross section measurements for $e^{-}+\mathrm{H}_{2} \mathrm{O}$ scattering, obtained with a high-angular resolution system, which (i) resolve earlier discrepancies among experiments, (ii) confirm a structure at intermediate energies for $\sigma_{T}$, and (iii) provide the first verification of theoretical predictions for $d \sigma_{e l} / d \Omega$ at any angle smaller than $10^{\circ}$.

The electrostatic positron beam at UCL [26], previously used to carry out measurements of $\sigma_{T}$ for $e^{+}+(\mathrm{He}, \mathrm{Kr})$ [27] and $e^{+}+\mathrm{H}_{2} \mathrm{O}$ [14] with an energy-independent discrimination $\theta \simeq\left(1-2^{\circ}\right)$, has been used to produce an electron beam with only slight modifications. Briefly, the secondary electrons, ejected from a tungsten mesh moderator [28] upon the impact of fast $\beta^{+}$particles emitted from ${ }^{22} \mathrm{Na}$ source, are accelerated to $2 \mathrm{keV}$ by applying a negative bias to the moderator and focused to a $\sim 1 \mathrm{~mm}$ radius spot onto a ( $W$-mesh) remoderator. A negative potential, $V_{R m}$, applied to the remoderator allows the beam 
energy to be varied according to $\left(E_{-}=e\left|V_{R m}\right|+\phi\right)$, where $\phi$ has been experimentally determined to be between 1.2-1.7 eV. The electrons are then transported round a cylindrical mirror analyzer to the interaction region, an $\mathrm{Al}$ cylindrical cell with aperture radius $=0.5 \mathrm{~mm}$ [27]. The electron beam has an angular divergence of $\simeq 1^{\circ}$ and an energy spread of $4 \%$. Its flight path is terminated by a position sensitive detector (PSD), which allows the reconstruction of the beam intensity distribution on the plane perpendicular to the beam axis.

The attenuation of the beam through the scattering cell is measured following the method of Ref. [14]. The total (elastic plus inelastic) cross sections are determined according to the Beer-Lambert law:

$$
\sigma_{T}=\frac{k_{B} T}{P l} \ln \left(\frac{I_{0}}{I}\right)
$$

where $I_{0}$ and $I$ are, respectively, the incident and transmitted beam intensity, $P$ and $T$ are the target gas pressure and temperature, respectively, $l$ is the length of the electron path through the gas, and $k_{B}$ is the Boltzmann constant. Periodically, a potential $\left(V_{R m}-9\right) \mathrm{V}$ is applied to a retarder grid in front of the detector to enable background measurements throughout the whole run. The same grid was biased at $V_{R m}$ during the beam-on measurements in order to discriminate against inelastically scattered particles.

The beam intensities $I$ and $I_{0}$ are extracted from different areas of the detector. In the present analysis, circular concentric domains around the beam spot center have been chosen and the radial profiles, $I(r)$ and $I_{0}(r)$, determined together with the profile $\sigma_{T}(r)$ for the total cross section, an example of which is given in Fig. 1. The beam center was taken at the maximum of the $I_{0}$ distribution [29], and an increment of $0.4 \mathrm{~mm}$ was used as a compromise between spatial (angular) resolution and statistical precision. The difference between $I(r)$ and $I_{0}(r)$ derives from the beam attenuation according to Eq. (1) and by the possible detection of FESP together with the transmitted beam $I$. The probability for this depends on the differential cross sections and the transfer function of the interaction region. Therefore, its contribution may change over the PSD area affecting the trend of $\sigma_{T}(r)$, as illustrated in Fig. 1. The detection probability of FESP versus scattering angle, computed for domains with $r=0.8 \mathrm{~mm}$ and $r=2.4 \mathrm{~mm}$, is shown in Fig. 2. The value $r=0.8 \mathrm{~mm}$ minimizes the contribution of forward scattered particles on our measurements whilst retaining sufficient precision, the values measured at $r=$ $0.4 \mathrm{~mm}$ possessing significantly higher statistical uncertainty due to the reduced detection area for this region. The estimated mean values of the angular acceptance, $\langle\theta\rangle$, are $\simeq 0.7^{\circ}$ and $\simeq 0.9^{\circ}$ for $0.8 \mathrm{~mm}$ and $2.4 \mathrm{~mm}$, respectively. Not all the particles scattered at $0^{\circ}$ can reach the detector because of the intrinsic divergence of the beam $\left(\simeq 1^{\circ}\right)[26]$. The detection of FESP produces artificially lower values of the attenuation

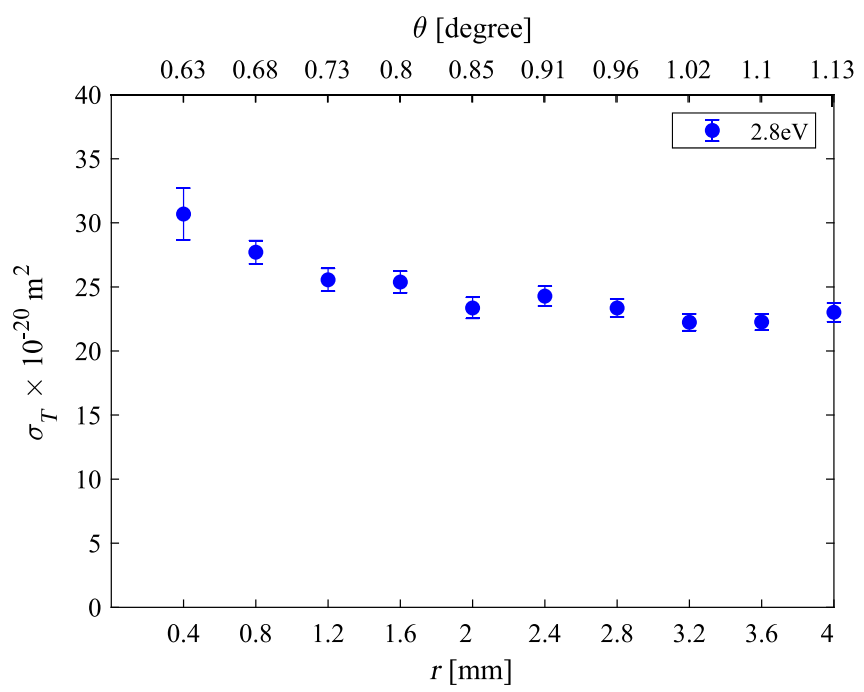

FIG. 1. Example of the radial dependence of the total cross section for $e^{-}+\mathrm{H}_{2} \mathrm{O}$ scattering at $2.8 \mathrm{eV}$. Top axis shows the angular acceptance corresponding to each value of $r$.

of the beam through the target, yielding smaller total cross sections. Thus, since $\theta$ increases with the radius, $\sigma_{T}(r)$ is a decreasing function of $r$.

The current results for the total cross sections for $e^{-}+\mathrm{H}_{2} \mathrm{O}$, taken from the radial profiles $\sigma_{T}(r)$ at $0.8 \mathrm{~mm}$, are shown in Figs. 3(a) and 3(b) together with previous experimental and theoretical determinations, respectively. Whilst a large spread of $\sigma_{T}$ values among previous measurements may be noted in Fig. 3(a) especially below $20 \mathrm{eV}$, a broad convergence emerges between the present results $\left(\langle\theta\rangle \simeq 0.7^{\circ}\right)$ and those of Ref. [30] for which $\theta \simeq 1.3^{\circ}$. However, at energies $\lesssim 6 \mathrm{eV}$, the present cross sections are $\simeq 30 \%$ higher.

In Fig. 3(b), the present $\sigma_{T}$ data are compared with theoretical predictions. Jain [33] used semiempirical

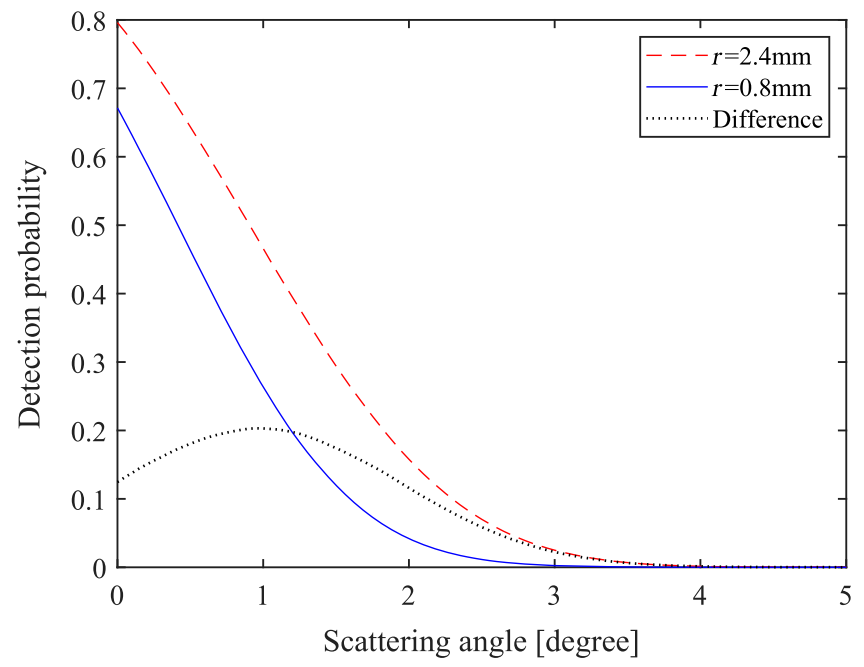

FIG. 2. Detection probability versus scattering angle for the current interaction region and for different radii at the PSD. 

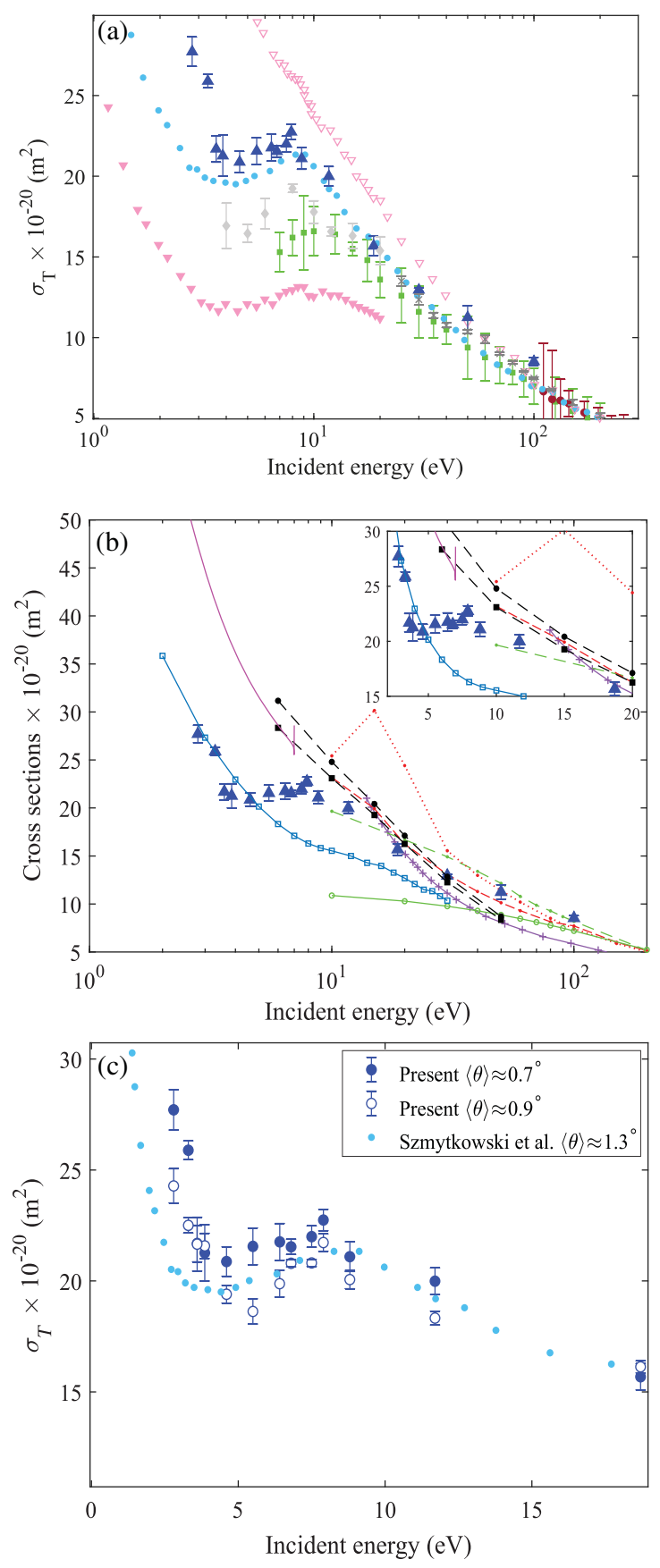

FIG. 3. (a) Total cross sections for electron scattering from $\mathrm{H}_{2} \mathrm{O}$. Solid symbols correspond to direct measurements, hollow symbols to measurements corrected for forward-angle elastic scattering: filled triangle present results, cyan filled circle [30], filled inverted triangle [15], open inverted triangle [13], filled square [16], darkred filled circle [31], times [32], filled rhombus [17]. (b) Total cross section comparison between present results filled triangle and theoretical predictions: dots SEP(CP)a1 [33], dotted dash SEP(ED) a1 [33]; integral elastic cross section dashed filled circle initial state $J=0$ [34], and dashed filled square thermal average [34]; dash additivity rule [35], dashed open circle semiempirical formula [35], dashed open square Schwinger approach [36], solid line $R$ matrix [37], dashed plus IAM plus BEB [30]. (c) Comparison among present $\sigma_{T}$ for $\langle\theta\rangle \simeq 0.7^{\circ}$ and $\langle\theta\rangle \simeq 0.9^{\circ}$, and results from Ref. [30]. spherical complex optical potentials to compute $\sigma_{T}$ over the energy range of 10-3000 $\mathrm{eV}$, recommending the $\mathrm{SEP}(\mathrm{CP})$ a1 model below $100 \mathrm{eV}$. Liu et al. [35] employed a semiempirical formula as well as the complex optical potential and the additivity rule to calculate $\sigma_{T}$ over an incident energy range of $10-1000 \mathrm{eV}$, the former being closer to experiment. Okamoto et al. [34] calculated vibrational elastic integral and differential cross sections using an $a b$ initio static potential and approximate electron exchange and target polarization. The Born closure formula was used for the evaluation of forward elastic scattering. The thermal averaged integral elastic cross section, including the contributions of the states with $J>0$ was shown to be $\leq 10 \%$ lower than the $J=0$ integral cross sections [34]. Both sets of results are shown in the figure. Varella et al. [36] applied the Schwinger multichannel method with pseudopotentials (combined with the Born closure procedure) to calculate the elastic cross section in the $2-30 \mathrm{eV}$ range. The estimations of Szmytkowski and Mozejko [30] are based on calculations of elastic and ionization cross sections using the independent atom method and binary encounter Bethe approach, respectively.

Faure et al. [37] investigated the vibrationally elastic and rotationally inelastic scattering of electrons at collision energies below $7 \mathrm{eV}$. The water molecule was described by an $a b$ initio multicentered wave function developed using the $R$-matrix method [22,38], while the scattering calculations were performed within the $\mathrm{FN}$ approximation (again corrected with the Born closure formula) (e.g., Refs. [23,24]) using the experimental value of the dipole moment (1.854D). The sharp rise around $7 \mathrm{eV}$ may be linked to a Feshbach resonance predicted to lie just below the first electronic state [38]. The rotational cross sections were computed for all transitions up to $J=5$ [37]. In making the comparison with present measurements, additional data have been computed in steps of $0.05^{\circ}$ for $d \sigma_{e l} / d \Omega$ [29]. Calculations were repeated as function of $J$ to check for the effect of temperature on $\sigma_{T}$. This was found to be small $(<10 \%)$ so only transitions from the initial state $J=0$ to paralevels are considered here. These calculations give excellent agreement with the very lowenergy $(\leq 0.25 \mathrm{eV})$ cross section of Curik et al. [39] whose experimental method recovers, but does not resolve, the low angle contribution to $\sigma_{T}$. Besides temperature effects, the major source of uncertainty in these calculations is the use of the fixed nuclei approximation that neglects the vibrational motion and relaxation of the molecule caused by the scattering electron.

The present experimental results corroborate the presence of a shoulder in the region (6-12 eV) where several resonances are known to occur. However, for instance, those related to dissociative attachment have much smaller cross sections $\left(10^{-22}-10^{-21} \mathrm{~m}^{2}\right)$ [7] than $\sigma_{T}$. The region $(6-8 \mathrm{eV})$ is also associated with vibrational excitation resonances. For example, the study of Ref. [20] found 
humps in the cross sections of maximum values $\left(\simeq 8 \times 10^{-21} \mathrm{~m}^{2}\right)$ for the combined $100+001$ stretching modes and $\left(\simeq 3 \times 10^{-21} \mathrm{~m}^{2}\right)$ for the bending 010 mode. Resonances associated with the electronically excited states of $\mathrm{H}_{2} \mathrm{O}$ have been predicted below $16 \mathrm{eV}$, the first lying just below the threshold at around $7 \mathrm{eV}[38,40]$. Measurements of the integral cross sections for excitation of the first six electronic states of $\mathrm{H}_{2} \mathrm{O}$ by $9-20 \mathrm{eV}$ electrons indicate values of $\left(\leq 10^{-21} \mathrm{~m}^{2}\right)[41,42]$.

The trend of $\sigma_{T}$ versus PSD radius has been studied to identify possible features ascribable to scattering at different angles. In Fig. 3(c), the values of $\sigma_{T}$ at $r=0.8 \mathrm{~mm}$ $\left(\langle\theta\rangle \simeq 0.7^{\circ}\right)$ are compared with the values at $r=2.4 \mathrm{~mm}$ $\left(\langle\theta\rangle \simeq 0.9^{\circ}\right)$. As expected, the values of $\sigma_{T}$ at intermediatelow energies are systematically lower for poorer angular discrimination.

The contribution of FESP to $\sigma_{T}$ can be written as:

$$
\Delta \sigma=2 \pi \int_{0^{\circ}}^{\theta_{\max }} d \sigma_{e l} / d \Omega \sin (\theta) F(\theta) d \theta,
$$

where $d \sigma_{e l} / d \Omega$ is the rotationally and vibrationally summed elastic differential cross section while $F(\theta)$ is the angular dependent detection probability with $F\left(\theta_{\max }\right)=0$. If we ascribe the difference between $\sigma_{T}$ at $\langle\theta\rangle \simeq 0.7^{\circ}$ and at $0.9^{\circ}$ to the contribution of FESP, then Eq. (2) together with the data shown in Fig. 3(c) may be used to estimate an average value of $d \sigma_{e l} / d \Omega$ in the angular range $\left(0, \theta_{\max }\right)$ as follows

$$
\left\langle d \sigma_{e l} / d \Omega\right\rangle \approx \frac{\sigma_{T}(0.8)-\sigma_{T}(2.4)}{2 \pi\left[\mathfrak{\Im}\left(\theta_{2.4}\right)-\mathfrak{I}\left(\theta_{0.8}\right)\right]},
$$

where $\sigma_{T}(0.8)$ and $\sigma_{T}(2.4)$ are the values of $\sigma_{T}(r)$ extracted at $r_{1}=0.8 \mathrm{~mm}$ and $r_{2}=2.4 \mathrm{~mm}$, respectively, and $\mathfrak{I}\left(\theta_{i}\right)=\int_{0^{\circ}}^{\theta_{i}} \sin (\theta) F_{i}(\theta) d \theta$. The values thus obtained for $\left\langle d \sigma_{e l} / d \Omega\right\rangle$ are shown in Fig. 4. There are no other experimental determinations of $d \sigma_{e l} / d \Omega$ near $1^{\circ}$ available for comparison. Indeed, as outlined in the introduction, the inherent difficulties in the discrimination of the scattered beam component at angles smaller than $10^{\circ}$ around the incident beam direction make these measurements extremely challenging $[19,21]$ and undetermined until now. In Ref. [29], the present experimental results are compared with the new $R$-matrix calculations using the method of Ref. [37]. However, as in Eq. (3), the experimental values are averages over the range of scattering angles $0-3.5^{\circ}$ with a mean scattering angle of $1.2^{\circ}$, as obtained from the distributions seen in Fig. 2. Thus the corresponding averages of the theoretical differential cross sections, $\left\langle d \sigma_{e l} / d \Omega\right\rangle_{T h}$, have been computed according to

$$
\left\langle d \sigma_{e l} / d \Omega\right\rangle_{T h}=\frac{1}{\mathfrak{s}\left(3.5^{\circ}\right)} \int_{0^{\circ}}^{3.5^{\circ}} d \sigma_{e l} / d \Omega \sin (\theta) F(\theta) d \theta,
$$

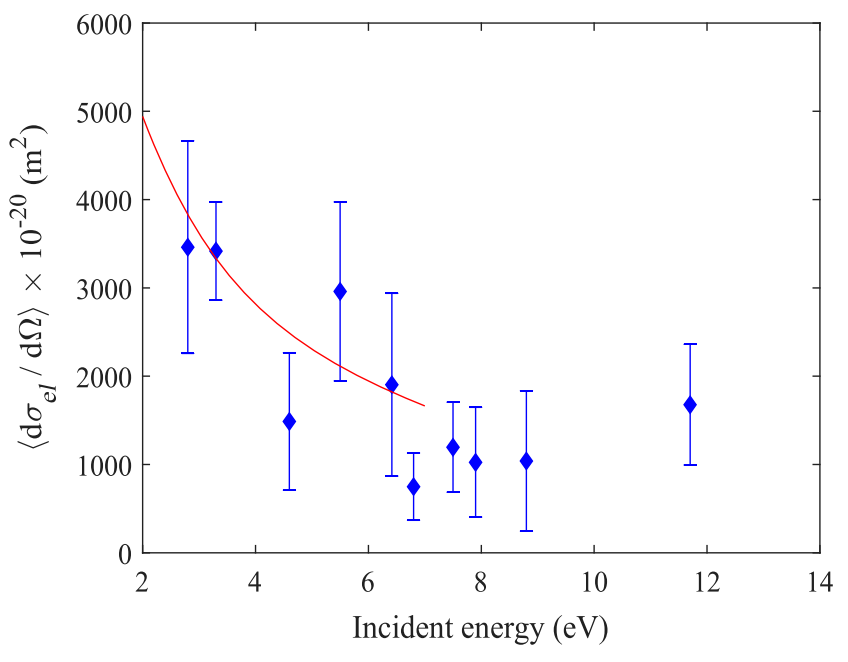

FIG. 4. Comparison between experimental $\left\langle d \sigma_{e l} / d \Omega\right\rangle$ (symbol) and theoretical $\left\langle d \sigma_{e l} / d \Omega\right\rangle_{T h}$ (line) at $\langle\theta\rangle \simeq 1.2^{\circ}$.

where the numerical integration of Eq. (4) has been performed using the trapezium rule. As illustrated in Fig. 4, most of the experimental values of $\left\langle d \sigma_{e l} / d \Omega\right\rangle$ are within one standard deviation of the corresponding theoretical results. Interestingly, a significant drop in $\left\langle d \sigma_{e l} / d \Omega\right\rangle$ may be noted in the region (6-9 eV) possibly due to coupling to inelastic channels which, as commented earlier, are rich of resonances in this region.

In conclusion, measurements of $\sigma_{T}$ for $e^{-}+\mathrm{H}_{2} \mathrm{O}$ scattering are presented for an angular acceptance of $\langle\theta\rangle \simeq 0.7^{\circ}$. At energies above $\simeq 7 \mathrm{eV}$, the present values confirm the results of Ref. [30], also characterized by a small $\theta$, both being 20-75\% higher than other experimental determinations, mostly obtained by using beams with a poorer angular discrimination. At energies below $\simeq 7 \mathrm{eV}$, the present $\sigma_{T}$ is $28-100 \%$ higher than all previous measurements confirming the intense forward-peaked behavior of $d \sigma_{e l} / d \Omega$ for $\mathrm{H}_{2} \mathrm{O}$. In addition to the angle-resolved measurements of $\sigma_{T}$, we report experimental estimations of the (rotationally and vibrationally summed) elastic differential cross section at around $1.2^{\circ}$, which provide a first point of contact with theories in an angular region crucially important for $\mathrm{H}_{2} \mathrm{O}$ and polar molecules in general.

The data supporting this publication are available at UCL Discovery [43].

We are grateful to The Engineering and Physical Sciences Research Council (UK) for supporting this work under Grant No. EP/P009395/1 and to John Dumper and Rafid Jawad for excellent technical assistance. A. K. thanks the Hungarian Scientific Research Foundation (OTKA Grant No. K128621).

[1] A. P. Gaiduk, T. A. Pham, M. Govoni, F. Paesani, and G. Gallii, Nat. Commun. 9, 247 (2018). 
[2] Y. Zhao et al., Proc. Natl. Acad. Sci. U.S.A. 109, 15612 (2012).

[3] M. Mucke, M. Braune, S. Barth, M. Förstel, T. Lischke, V. Ulrich, T. Arion, U. Becker, A. Bradshaw, and U. Hergenhahn, Nat. Phys. 6, 143 (2010).

[4] C. Miyake, Plant Cell Physiol. 51, 1951 (2010).

[5] W. Zheng, R. Jewitt, and D. Kaiser, Astrophys. J. 639, 534 (2006).

[6] P. Bernath, Phys. Chem. Chem. Phys. 4, 1501 (2002).

[7] Y. Itikawa and N. Mason, J. Phys. Chem. Ref. Data 34, 1 (2005).

[8] G. Garcia and M. Fuss, Radiation Damage in Biomolecular Systems (Springer, London, 2012), pp. 1-506.

[9] M. Brunger, Int. Rev. Phys. Chem. 36, 333 (2017).

[10] G. Diniz, C. Rutjes, U. Ebert, and I. Ferreira, J. Geophys. Res. 124, 189 (2019).

[11] S. Incerti et al., Med. Phys. 45, e722 (2018).

[12] B. Boudaïffa, P. Cloutier, D. Hunting, M. Huels, and L. Sanche, Science 287, 1658 (2000).

[13] M. Kimura, O. Sueoka, A. Hamada, and Y. Itikawa, A Comparative Study of Electron-and Positron-Polyatomic Molecule Scattering (John Wiley \& Sons, Inc., New York, 2000), pp. 537-622.

[14] A. Loreti, R. Kadokura, S. E. Fayer, A. Kövér, and G. Laricchia, Phys. Rev. Lett. 117, 253401 (2016).

[15] O. Sueoka, S. Mori, and Y. Katayama, J. Phys. B 19, L373 (1986).

[16] H. Nishimura and K. Yano, J. Phys. Soc. Jpn. 57, 1951 (1988).

[17] Z. Saglam and N. Aktekin, J. Phys. B 24, 3491 (1991).

[18] R. Zhang, A. Faure, and J. Tennyson, Phys. Scr. 80, 015301 (2009).

[19] H. Y. Cho, Y. S. Park, H. Tanaka, and S. Buckman, J. Phys. B 37, 625 (2004).

[20] M. A. Khakoo, C. Winstead, and V. McKoy, Phys. Rev. A 79, 052711 (2009).

[21] M. A. Khakoo, H. Silva, J. Muse, M. C. A. Lopes, C. Winstead, and V. McKoy, Phys. Rev. A 87, 049902(E) (2013).

[22] J. Tennyson, Phys. Rep. 491, 29 (2010).
[23] F. Gianturco and S. Scialla, J. Chem. Phys. 87, 6468 (1987).

[24] F. A. Gianturco and A. Jain, Phys. Rep. 143, 347 (1986).

[25] I. I. Fabrikant, J. Phys. B 49, 222005 (2016).

[26] A. Kövér, A. I. Williams, D. J. Murtagh, S. E. Fayer, and G. Laricchia, Meas. Sci. Technol. 25, 075013 (2014).

[27] S. Fayer, A. Loreti, S. Andersen, A. Kövér, and G. Laricchia, J. Phys. B 49, 075202 (2016).

[28] A. I. Williams, D. J. Murtagh, S. E. Fayer, S. L. Andersen, J. Chevallier, Kövér, P. V. Reeth, J. W. Humberston, and G. Laricchia, J. Appl. Phys. 118, 105302 (2015).

[29] See Supplemental Material at http://link.aps.org/ supplemental/10.1103/PhysRevLett.123.033401 for an image of the beam spot at the PSD and for the $R$-matrix calculations of the differential elastic cross sections between 0-3 degree at 2.8, 4.6 and $6.8 \mathrm{eV}$.

[30] C. Szmytkowski and P. Mozejko, Optica Applicata 36, 543 (2006).

[31] A. Zecca, G. Karwasz, S. Oss, R. Grisenti, and R. S. Brusa, J. Phys. B 20, L133 (1987).

[32] Z. Saglam and N. Aktekin, J. Phys. B 23, 1529 (1990).

[33] A. Jain, J. Phys. B 21, 905 (1988).

[34] Y. Okamoto, K. Onda, and Y. Itikawa, J. Phys. B 26, 745 (1993).

[35] Y. Liu, J. Sun, Z. Li, Y. Juang, and L. Wan, Z. Phys. D 42, 45 (1997).

[36] M. Varella, M. Bettega, M. Lima, and L. Ferreira, J. Chem. Phys. 111, 6396 (1999).

[37] A. Faure, J. D. Gorfinkiel, and J. Tennyson, Mon. Not. R. Astron. Soc. 347, 323 (2004).

[38] J. D. Gorfinkiel, L. A. Morgan, and J. Tennyson, J. Phys. B 35, 543 (2002).

[39] R. Curik, J. P. Ziesel, N. C. Jones, T. A. Field, and D. Field, Phys. Rev. Lett. 97, 123202 (2006).

[40] T. N. Rescigno and A. E. Orel, Phys. Rev. A 88, 012703 (2013).

[41] K. Ralphs, G. Serna, L. R. Hargreaves, M. A. Khakoo, C. Winstead, and V. McKoy, J. Phys. B 46, 125201 (2013).

[42] M. Matsui, M. Hoshino, H. Kato, F. F. da Silva, P. LimaoVieira, and H. Tanaka, Eur. Phys. J. D 70, 77 (2016).

[43] See https://doi.org/10.14324/000.ds.10074515. 